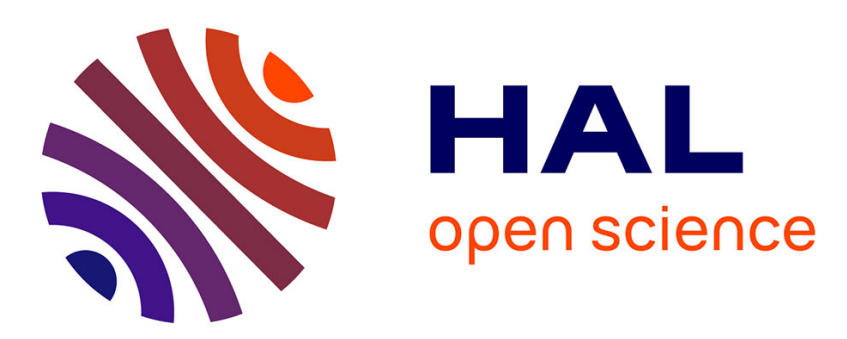

\title{
Infection in a dish: high-throughput analyses of bacterial pathogenesis.
}

C. Léopold Kurz, Jonathan J. Ewbank

\section{To cite this version:}

C. Léopold Kurz, Jonathan J. Ewbank. Infection in a dish: high-throughput analyses of bacterial pathogenesis.. Current Opinion in Microbiology, 2007, 10 (1), pp.10-16. 10.1016/j.mib.2006.12.001 . inserm-00122557

\section{HAL Id: inserm-00122557 https://www.hal.inserm.fr/inserm-00122557}

Submitted on 3 Jan 2007

HAL is a multi-disciplinary open access archive for the deposit and dissemination of scientific research documents, whether they are published or not. The documents may come from teaching and research institutions in France or abroad, or from public or private research centers.
L'archive ouverte pluridisciplinaire HAL, est destinée au dépôt et à la diffusion de documents scientifiques de niveau recherche, publiés ou non, émanant des établissements d'enseignement et de recherche français ou étrangers, des laboratoires publics ou privés. 


\section{HAL author manuscript}

Infection in a dish: high-throughput analyses of bacterial pathogenesis

C. Léopold Kurz ${ }^{1,2,3}$ \& Jonathan J. Ewbank ${ }^{1,2,3, *}$

${ }^{1}$ Centre d'Immunologie de Marseille-Luminy, Université de la Méditerranée, Case 906, 13288

Marseille cedex 9, France

${ }^{2}$ INSERM, U631, 13288 Marseille, France

${ }^{3}$ CNRS, UMR6102, 13288 Marseille, France

*Correspondence: ewbank@ciml.univ-mrs.fr

Running head: Infection in a dish

\section{SUMMARY}

Diverse aspects of host-pathogen interactions have been studied using non-mammalian hosts such as Dictyostelium discoideum, Caenorhabditis elegans, Drosophila melanogaster and Danio rerio for more than 20 years. Over the last two years, the use of these model hosts to dissect bacterial virulence mechanisms has been expanded to include the important human pathogens Vibrio cholerae and Yersinia pestis. Innovative approaches using these alternative hosts have also been developed, allowing new antimicrobials to be isolated through screening large libraries of compounds in a C. elegans-Staphylococcus aureus infection model. Host proteins needed by Mycobacterium and Listeria during their invasion and intracellular growth have been uncovered by high-throughput dsRNA screens in a Drosophila cell culture system, while immune evasion mechanisms deployed by Pseudomonas aeruginosa during its infection of flies have been identified. Together, these reports further illustrate the potential and relevance of these non-mammalian hosts for modelling diverse aspects of bacterial infection in mammals. 


\section{INTRODUCTION}

The use of mammalian models to identify and understand virulence factors of human pathogens is indispensable. Alternative models, such as the amoeba Dictyostelium discoideum, the nematode Caenorhabditis elegans, the insect Drosophila melanogaster and the fish Danio rerio are, however, complementary systems for such studies [1-5]. This is possible because many human pathogens are of low species specificity and can infect hosts ranging from insects and nematodes to fish, as well as other mammals. They rely on universal virulence factors that are involved in the infection process regardless of the host. These can be identified and characterised using genetically tractable and inexpensive non-mammalian models. In addition, the molecular and genetic tools developed with these simple organisms combined with their well-studied cellular biology and/or immunology allow one to decipher the complex interactions between host and pathogen.

The four organisms listed above have in common many characteristics, such as ease of culture and full sequenced genomes, that make them very useful as model hosts [1]. These alternative hosts are being used for approaches as diverse as testing the virulence of chosen pathogen mutants $[6, * 7]$, screening large banks of pathogen mutants for those with attenuated virulence $[8, * 9, * 10]$ or dissecting the host mechanisms involved in pathogen invasion and intracellular replication $[* * 11, * 12, * 13]$.

In addition, they have unique features relevant to the study of specific aspects of hostpathogen interactions. The amoeba $D$. discoideum is a professional phagocyte that can be used to decipher the molecular basis of phagocytosis and phagosome maturation [4]. Additionally, it can give insights into how certain intracellular bacterial pathogens survive in the phagolysosome [14]. The fly D. melanogaster possesses a very well-studied innate immunity [15] that has contributed to the understanding of immune mechanisms in mammals. More 
recently, it has been used to analyze the mechanisms used by pathogens to evade the host immune system $[* 16,17, * * 18]$. Finally, genetic screens for bacterial virulence genes in a vertebrate with a fully developed immune system [19] are possible with the fish D. rerio. This review focuses on recent work with the alternative model hosts D. discoideum, C. elegans, D. melanogaster and D. rerio in these new investigative paradigms.

\section{New infections modelled with alternative hosts.}

An increasing number of human bacterial pathogens are being tested in non-mammalian hosts in order to study conveniently their virulence. In addition to established models such as Pseudomonas aeruginosa [20,21], Salmonella typhimurium [22-24] or Serratia marcescens $[25,26]$, several pathogens including Listeria monocytogenes [27,28], Yersinia pestis (see below) and Vibrio cholerae, the causal agent of cholera, have recently been added to the list of micro-organisms able to cause lethal infection of the nematode and the fly.

In humans, expression of Cholera toxin (CT) by $V$. cholerae provokes a rise in cAMP in the intestinal epithelium, the opening of ion channels and consequently loss of water into the intestinal lumen. In mice, this secretory diarrhea can be successfully treated with the channel blocker clotrimazole. It has now been reported that oral $V$. cholerae infection of the fruit fly leads to the death of the animals in a manner somewhat similar to that observed in humans, including rapid weight loss [*7]. CT is required for full virulence in the fly model and, remarkably, flies with loss-of-function mutations in genes encoding homologues of the known targets of CT resist infection. Furthermore, clotrimazole can help cure flies infected with $V$. cholerae [*7].

During the lethal colonization of the $C$. elegans intestine by $V$. cholerae, on the other hand, CT does not appear to play an important role [6]. But, using a reverse genetic approach, Vaitkevicius and colleagues demonstrated that the quorum sensing regulated protease PrtV is 
essential for this killing. Moreover, they obtained data [6] suggesting that this protease is important for $V$. cholerae in its natural niche [29] for its resistance to the marine plankton that graze on it. Finally, they measured an increased IL-8 secretion in human epithelial intestinal cells exposed to a $V$. cholerae prt $V$ deletion mutant, compared to the parental strain, suggesting a role for this protease in modulating (directly or indirectly) the host response in vertebrates [6].

Together, these reports illustrate to what extent nematode and fly can be relevant for the study of the causative agent of cholera. Importantly, the work by Blow and colleagues offers the perspective of using Drosophila to screen for chemicals that inhibit CT in vivo, following a precedent set by the Ausubel lab using C. elegans [**30].

\section{In vivo screens for new antimicrobials}

The massive use of antibiotics combined with the high adaptation capacity of bacteria has created the public-health problem of human pathogens resistant to multiple antibiotics. Therefore, there is a real need for new antibiotic molecules. Moy and colleagues cleverly used an infection system involving a C. elegans immunocompromised mutant and Enterococcus faecalis $[* * 30]$ to screen for synthetic $(6,000)$ and natural $(>1,000)$ molecules that promoted host survival (FIGURE 1). This in vivo screen not only permitted the identification of 8 molecules affecting bacterial growth in vitro (MIC $<35 \mu \mathrm{g} / \mathrm{ml}$ ), but also of 8 other products either impairing pathogen virulence or boosting host innate immunity in the absence of significant in vitro activity $(\mathrm{MIC}>125 \mu \mathrm{g} / \mathrm{ml})$ [**30]. Even though the efficiency and toxicity of the identified molecules does need to be tested in mammals, this system represents a very promising screening platform to identify in vivo new antibacterial molecules. A similar system involving flies to identify antifungal drugs is also being developed $[31,32]$.

\section{Random screens for the identification of bacterial virulence genes}


Three recent reports using D. discoideum, C. elegans, and D. rerio as hosts to screen bacterial mutant libraries of Klebsiella pneumoniae, Y. pestis, and Streptococcus iniae, respectively, have further strengthened the relevance of these simple hosts.

K. pneumoniae is an important human pathogen that, as its name suggests, causes pneumonia. Its interaction with alveolar macrophages can be modelled using $D$. discoideum as a surrogate phagocyte. D. discoideum is normally able to feed on wild-type Klebsiella. Benghezal and colleagues elegantly combined the genetics of $D$. discoideum and the genetics of $K$. pneumoniae. They first identified a new gene (phgl) [33] rendering the amoeba especially susceptible to infection and unable to grow on Klebsiella. Then, they isolated Klebsiella mutants that supported the growth of the phgl mutant amoeba [*10]. Among the mutated bacterial genes were several required for LPS and amino-acid biosynthesis. They tested several of the isolated bacterial mutants in a mouse pneumonia model and found an attenuation of virulence [*10].

The genetic manipulation of both host and pathogen allowed them to create a $2 \mathrm{D}$ virulence array showing that distinct groups of host genes are necessary to resist infection by various bacterial pathogens and mutants (FIGURE 2). They were also able to demonstrate conservation of both virulence factors and defence genes since Drosophila phgl mutants are more susceptible to K. pneumoniae infection [*10].

Y. pestis, the causative agent of plague, can form a biofilm that is important for its dissemination via its vector, the flea. A Y. pestis biofilm can also accumulate on the head of C. elegans, and this is clearly a more accessible model to study biofilm function than looking in the gut of the flea [34]. But as biofilm formation is only one aspect of $Y$. pestis pathogenicity, Styer and colleagues developed a nematode-based infection system to identify $Y$. pestis virulence genes not related to biofilm formation [*9]. They showed that a biofilmdeficient mutant of $Y$. pestis colonises the intestine of $C$. elegans and provokes an early death. 
They used this infection model to screen a bank of $Y$. pestis mutants for those with attenuated virulence in the worm. Remarkably, despite the differences between nematodes and mammals, they identified two genes whose products are necessary for induction of mouse death in an intranasal mouse model of $Y$. pestis pathogenesis, genes that had previously not been implicated in $Y$. pestis virulence [*9].

S. iniae is a bacterial pathogen able to infect fish and humans. In order to analyze the interaction between streptococcal pathogens and their natural hosts, Miller and colleagues created a bank of bacterial mutants and screened it using zebrafish [8]. They wished to identify bacterial mutants specifically deficient in their capacity to disseminate in the brain. To facilitate the screening process, they used a Signature-Tagged Mutagenesis strategy [35] that permitted the analysis of fish co-infected by a pool of 12 distinct mutant strains. Doing so, they screened 1,128 signature-tagged transposon bacterial mutants and determined which bacterial mutants were not present in brain extracts from infected fish. Interestingly, 7 out of the 41 bacterial mutants isolated had transposon insertions in genes potentially coding for surface polysaccharides, major components of the capsule. Finally, using the bacterial mutants they isolated, they showed in a human whole blood assay for phagocytosis that $S$. iniae's capsule is involved in invasion and survival in human macrophages [8].

These three studies further validate the use of non-mammalian hosts for large-scale screens to identify bacterial virulence genes relevant to infection in mammals. Moreover, the genetic manipulation of the host as exemplified by the work of Benghezal et al., expands the range of models available for this kind of screening approach, in a manner reminiscent of the directed modification of mice, via trangenesis [36] or the creation of human-mouse chimeras [37], but without any of the ethical concerns.

Identification of host molecules required for pathogenesis and how the pathogen evades the immune system. 
The host factors involved in the infection processes are not restricted to "immunity" genes such as those coding for interleukins or Toll-like receptors (TLRs). This is especially the case for intracellular bacterial pathogens that have to enter the cell and avoid being degraded in phagolysosomes. Therefore, intracellular bacteria have developed many ways to hijack the endocytic or phagocytic routes [38,39]. Macrophages are often confronted by intracellular pathogens as they are professional phagocytes. Three recent studies used the Drosophila S2 macrophage-like cell line to perform large scale RNAi screens to identify host factors required for entry and survival of intracellular bacterial pathogens.

The first two analyses combined automated microscopy with the use of GFP-tagged Mycobacterium fortuitum [*13] or Listeria monocytogenes [*12] to screen a bank containing 21,300 dsRNAs (targeting $>95 \%$ of annotated Drosophila genes in a redundant fashion). They showed that factors involved in vesicle trafficking and actin cytoskeleton organization are necessary for internalization and intracellular survival of these two pathogens. Moreover, they identified a Drosophila homologue of the scavenger receptor CD36 as being crucial for $L$. monocytogenes and $M$. fortuitum entry into the S2 cells, while being dispensable for phagocytosis in general $\left[* 12,{ }^{*} 13\right]$. Based on these observations, the study was extended to mammalian cells and new roles in bacteria uptake described for members of the CD36 family. This work also highlighted a role for autophagy in the control of L. monocytogenes infection $[* 12]$

In contrast to these two studies that used automated microscopy, a third study was performed manually [**11]. In this painstaking project, interest was focused especially on the interaction between the L. monocytogenes toxin listeriolysin O (LLO) and host factors that allow the bacteria to escape from the phagosome. The authors combined RNAi against the host with bacterial mutants for LLO. In a first set of experiments, they used an LLO-deficient bacterial strain and screened for dsRNAs that restored the capacity of these mutants to escape 
into the cytoplasm. The corresponding genes would be expected to be elements of the host pathways targeted by LLO. In a second set of experiments, they used a bacterial mutant producing a LLO toxin lacking a PEST sequence (and thus with a longer half-life). They screened for dsRNAs that rendered S2 cells more susceptible to this stable toxin to determine which host enzymes control LLO toxicity. Based on their results, they propose a model in which the pore-forming LLO inserts into the membrane of the L. monocytogenes-containing phagosome, thus impairing its acidification and maturation. Concerning the host's control of LLO toxicity, their screen identified SPT, an enzyme necessary for sphingolipid metabolism, as a key factor for host resistance [**11].

The experimental systems described in these three reports can thus be used to shed light on the complex interactions between the host and an intracellular pathogen that are both fighting for their survival. But just as is the case for any model system, the results come with a number of caveats. It is well known that a dsRNA can interfere with off-target genes and so generate false positive results [40]. And conversely, important genes can be missed if they are not expressed in or on S2 cells, as is indeed the case for some receptors involved in phagocytosis (Istvan Ando and Dan Hultmark, personal communication). Nevertheless, in the long term, by combining large-scale screens in the host and the pathogen, it will be possible to define a host-pathogen interactome (Figure 2) [41].

Extracellular bacterial pathogens are usually not able to survive phagocytosis. Many, however, have developed strategies to counteract the humoral arm of the host immune system. A handful of recent articles have demonstrated that Pseudomonas infection of D. melanogaster is a most suitable system to study the host immune response and to uncover the strategies used by the pathogen to elude defence mechanisms. In one article, the role of the Pseudomonas exotoxin ExoS was directly addressed by ectopic expression in the eye or by ubiquitous expression of this bacterial protein in the fly [17]. The authors showed with 
these transgenic systems that ExoS inhibits the activity of a host RhoGTPase in vivo and that ubiquitous ExoS expression impairs the phagocytic capacity of fly macrophages without affecting anti-microbial peptide gene induction [17]. In a complementary study, Liehl and colleagues used host and pathogen mutants to demonstrate that the Pseudomonas AprA metalloprotease directly degrades fly antimicrobial peptides [**18]. This protease thereby acts as a virulence factor by enhancing bacterial survival within the host body fluid. In addition to these reports, Apidianakis and colleagues compared microarray results from flies infected by virulent or avirulent $P$. aeruginosa strains [*16]. Strikingly, this analysis revealed an as yet uncharacterised mechanism used by $P$. aeruginosa in the early phases of the infection to limit Drosophila antimicrobial genes expression at the transcriptional level.

Together, these studies illustrate the potential use of genetically tractable non-mammalian hosts, with characterized immune systems, to decipher the mechanisms pathogens employ to evade the host immune system. As exemplified above, it is possible to have a global approach and/or to address precisely the role of a specific bacterial protein.

\section{CONCLUSIONS}

Molecular and physiological conservation within different bacteria and across eukaryotic species renders the study of host-pathogen interaction using non-mammalian models especially attractive. Moreover, it is likely that many of the virulence mechanisms that pathogens use during their infection of humans in fact evolved since they favour survival in the natural ecological niche and so are best studied using their natural predators, such as D. discoideum and C. elegans. After a period when these model systems were used in essentially one-sided approaches (e.g. screening banks of bacterial mutants for virulence genes or identifying the host targets of bacterial virulence factors), more and more studies are now exploiting a combination of bacterial and host genetics to address the molecular basis of 
pathogenicity and defence. The future promises to reveal details of the intimate but deadly dance between pathogen and host that has been going on since the birth of eukaryotes. 


\section{REFERENCES}

1. Steinert M, Heuner K: Dictyostelium as host model for pathogenesis. Cell Microbiol $2005,7: 307-314$.

2. Sifri CD, Begun J, Ausubel FM: The worm has turned--microbial virulence modeled in Caenorhabditis elegans. Trends Microbiol 2005, 13:119-127.

3. Vodovar N, Acosta C, Lemaitre B, Boccard F: Drosophila: a polyvalent model to decipher host-pathogen interactions. Trends Microbiol 2004, 12:235-242.

4. van der Sar AM, Musters RJ, van Eeden FJ, Appelmelk BJ, Vandenbroucke-Grauls CM, Bitter W: Zebrafish embryos as a model host for the real time analysis of Salmonella typhimurium infections. Cell. Microbiol. 2003, 5:601-611.

5. Pradel E, Ewbank JJ: Genetic models in pathogenesis. Annu Rev Genet 2004, 38:347-363.

6. Vaitkevicius K, Lindmark B, Ou G, Song T, Toma C, Iwanaga M, Zhu J, Andersson A, Hammarstrom ML, Tuck S, et al.: A Vibrio cholerae protease needed for killing of Caenorhabditis elegans has a role in protection from natural predator grazing. Proc Natl Acad Sci U S A 2006, 103:9280-9285.

*7. Blow NS, Salomon RN, Garrity K, Reveillaud I, Kopin A, Jackson FR, Watnick PI:

Vibrio cholerae infection of Drosophila melanogaster mimics the human disease cholera. PLoS Pathog 2005, 1:e8.

This report establishes $D$. melanogaster as a new in vivo model to study $V$. cholerae infection. As for mammals, the infection is per os and CT is essential. Moreover, the host machinery involved in the subsequent secretory diarrhea is conserved.

8. Miller JD, Neely MN: Large-scale screen highlights the importance of capsule for virulence in the zoonotic pathogen Streptococcus iniae. Infect Immun 2005, 73:921934. 
*9. Styer KL, Hopkins GW, Bartra SS, Plano GV, Frothingham R, Aballay A: Yersinia pestis kills Caenorhabditis elegans by a biofilm-independent process that involves novel virulence factors. $E M B O$ Rep 2005, 6:992-997.

The authors demonstrate that $Y$. pestis can infect $C$. elegans in a biofilm-independent fashion and use this infection model to identify new $Y$. pestis factors that are also necessary for full virulence in mice.

*10. Benghezal M, Fauvarque MO, Tournebize R, Froquet R, Marchetti A, Bergeret E, Lardy B, Klein G, Sansonetti P, Charette SJ, et al.: Specific host genes required for the killing of Klebsiella bacteria by phagocytes. Cell Microbiol 2006, 8:139-148.

In this study, $D$. discoideum and $K$. pneumoniae genetics were combined to identify host resistance genes and to screen for pathogen virulence factors, respectively. Importantly, the bacterial virulence genes identified are necessary for full-killing in a mouse pneumonia model.

**11. Cheng LW, Viala JP, Stuurman N, Wiedemann U, Vale RD, Portnoy DA: Use of RNA interference in Drosophila $\mathrm{S} 2$ cells to identify host pathways controlling compartmentalization of an intracellular pathogen. Proc Natl Acad Sci U S A 2005, 102:13646-13651.

The authors used a bank of 7,216 dsRNA to identify host factors involved in pathogenesis of Drosophila S2 cells infected by L. monocytogenes. In order to uncover the host pathways targeted by the bacterial LLO toxin and host mechanisms protecting the cell from this toxin, they combined this large-scale approach with bacterial mutants. They identified host proteins not previously involved in Listeria pathogenesis and enzymes involved in LLO-detoxification. 
*12. Agaisse H, Burrack LS, Philips JA, Rubin EJ, Perrimon N, Higgins DE: Genome-wide RNAi screen for host factors required for intracellular bacterial infection. Science 2005, 309:1248-1251.

*13. Philips JA, Rubin EJ, Perrimon N: Drosophila RNAi screen reveals CD36 family member required for mycobacterial infection. Science 2005, 309:1251-1253.

In order to uncover host factors necessary for intracellular bacterial infection, these two papers used genome-wide RNAi bank, a Drosophila macrophage-like cell line, automated microscopy and GFP-tagged L. monocytogenes [*12] or M. fortiutum.[*13] Several hundred dsRNA altering the progression of infection were identified. The results obtained in the two screens were directly compared [*12]. Peste, a member of the CD36 family was found to be crucial for the entry of both bacteria into cells.

14. Li Z, Solomon JM, Isberg RR: Dictyostelium discoideum strains lacking the RtoA protein are defective for maturation of the Legionella pneumophila replication vacuole. Cell Microbiol 2005, 7:431-442.

15. Royet J, Reichhart JM, Hoffmann JA: Sensing and signaling during infection in Drosophila. Curr Opin Immunol 2005, 17:11-17.

*16. Apidianakis Y, Mindrinos MN, Xiao W, Lau GW, Baldini RL, Davis RW, Rahme LG: Profiling early infection responses: Pseudomonas aeruginosa eludes host defenses by suppressing antimicrobial peptide gene expression. Proc Natl Acad Sci U S A 2005, 102:2573-2578.

In this study, the authors performed microarray analyses on flies infected with either wild-type $P$. aeruginosa or an avirulent mutant. Strikingly, they uncovered an enigmatic mechanism used by $P$. aeruginosa to silence specifically the expression of genes encoding antimicrobial proteins. 
17. Avet-Rochex A, Bergeret E, Attree I, Meister M, Fauvarque MO: Suppression of Drosophila cellular immunity by directed expression of the ExoS toxin GAP domain of Pseudomonas aeruginosa. Cell Microbiol 2005, 7:799-810.

**18. Liehl P, Blight M, Vodovar N, Boccard F, Lemaitre B: Prevalence of local immune response against oral infection in a Drosophila/Pseudomonas infection model. PLoS Pathog 2006, 2:e56.

The authors developed an oral infection model between Drosophila and a newly identified entomopathogen $P$. entomophila and identified and characterised a bacterial metalloprotease abrogating host defences through degradation of antimicrobial peptides.

19. Trede NS, Langenau DM, Traver D, Look AT, Zon LI: The use of zebrafish to understand immunity. Immunity 2004, 20:367-379.

20. D'Argenio DA, Gallagher LA, Berg CA, Manoil C: Drosophila as a model host for Pseudomonas aeruginosa infection. J. Bacteriol. 2001, 183:1466-1471.

21. Tan MW, Mahajan-Miklos S, Ausubel FM: Killing of Caenorhabditis elegans by Pseudomonas aeruginosa used to model mammalian bacterial pathogenesis. Proc. Natl. Acad. Sci. USA 1999, 96:715-720.

22. Brandt SM, Dionne MS, Khush RS, Pham LN, Vigdal TJ, Schneider DS: Secreted Bacterial Effectors and Host-Produced Eiger/TNF Drive Death in a SalmonellaInfected Fruit Fly. PLoS Biol 2004, 2:e418.

23. Aballay A, Yorgey P, Ausubel FM: Salmonella typhimurium proliferates and establishes a persistent infection in the intestine of Caenorhabditis elegans. Curr. Biol. 2000, 10:1539-1542.

24. Labrousse A, Chauvet S, Couillault C, Kurz CL, Ewbank JJ: Caenorhabditis elegans is a model host for Salmonella typhimurium. Curr Biol 2000, 10:1543-1545. 
25. Flyg C, Kenne K, Boman HG: Insect pathogenic properties of Serratia marcescens: phage-resistant mutants with a decreased resistance to Cecropia immunity and a decreased virulence to Drosophila. J. Gen. Microbiol. 1980, 120:173-181.

26. Kurz CL, Chauvet S, Andres E, Aurouze M, Vallet I, Michel GP, Uh M, Celli J, Filloux A, De Bentzmann S, et al.: Virulence factors of the human opportunistic pathogen Serratia marcescens identified by in vivo screening. EMBO J. 2003, 22:1451-1460.

27. Thomsen LE, Slutz SS, Tan MW, Ingmer H: Caenorhabditis elegans is a model host for Listeria monocytogenes. Appl Environ Microbiol 2006, 72:1700-1701.

28. Mansfield BE, Dionne MS, Schneider DS, Freitag NE: Exploration of host-pathogen interactions using Listeria monocytogenes and Drosophila melanogaster. Cell. Microbiol. 2003, 5:901-911.

29. Reidl J, Klose KE: Vibrio cholerae and cholera: out of the water and into the host. FEMS Microbiol Rev 2002, 26:125-139.

**30. Moy TI, Ball AR, Anklesaria Z, Casadei G, Lewis K, Ausubel FM: Identification of novel antimicrobials using a live-animal infection model. Proc Natl Acad Sci U S A 2006, 103:10414-10419. Using a C. elegans-E. faecalis infection model, the authors developed a high-throughput platform to identify antimicrobial compounds in vivo. Interestingly, the majority of the identified molecules are not antimicrobial in vitro, but only efficient in vivo. This suggests that they may either boost the host immune system or act via the neutralization of some bacterial virulence factors.

31. Lionakis MS, Kontoyiannis DP: Fruit flies as a minihost model for studying drug activity and virulence in Aspergillus. Med Mycol 2005, 43 Suppl 1:S111-114.

32. Chamilos G, Lionakis MS, Lewis RE, Lopez-Ribot JL, Saville SP, Albert ND, Halder G, Kontoyiannis DP: Drosophila melanogaster as a facile model for large-scale studies 
of virulence mechanisms and antifungal drug efficacy in Candida species. $J$ Infect Dis 2006, 193:1014-1022.

33. Cornillon S, Pech E, Benghezal M, Ravanel K, Gaynor E, Letourneur F, Bruckert F, Cosson P: Phg1p is a nine-transmembrane protein superfamily member involved in Dictyostelium adhesion and phagocytosis. J Biol Chem 2000, 275:34287-34292.

34. Darby C, Hsu JW, Ghori N, Falkow S: Caenorhabditis elegans: plague bacteria biofilm blocks food intake. Nature 2002, 417:243-244.

35. Hensel M, Shea JE, Gleeson C, Jones MD, Dalton E, Holden DW: Simultaneous identification of bacterial virulence genes by negative selection. Science 1995, 269:400-403.

36. Lecuit M, Cossart P: Genetically-modified-animal models for human infections: the Listeria paradigm. Trends Mol Med 2002, 8:537-542.

37. Legrand N, Weijer K, Spits H: Experimental models to study development and function of the human immune system in vivo. J Immunol 2006, 176:2053-2058.

38. Henry T, Gorvel JP, Meresse S: Molecular motors hijacking by intracellular pathogens. Cell Microbiol 2006, 8:23-32.

39. Ismail N, Olano JP, Feng HM, Walker DH: Current status of immune mechanisms of killing of intracellular microorganisms. FEMS Microbiol Lett 2002, 207:111-120.

40. Kulkarni MM, Booker M, Silver SJ, Friedman A, Hong P, Perrimon N, Mathey-Prevot B: Evidence of off-target effects associated with long dsRNAs in Drosophila melanogaster cell-based assays. Nat Methods 2006, 3:833-838.

41. Tenor JL, McCormick BA, Ausubel FM, Aballay A: Caenorhabditis elegans-based screen identifies Salmonella virulence factors required for conserved hostpathogen interactions. Curr Biol 2004, 14:1018-1024. 


\section{FIGURES}

\section{Figure 1}

Protocol used by Moy and colleagues [**30] to screen in vivo for new antimicrobial compounds using an established C. elegans-E. faecalis infection system. After culture and amplification of nematode numbers on growth plates (seeded with the Escherichia coli strain OP50) synchronised populations of worms are transferred to infection plates, seeded with E. faecalis strain MMH594. After 8 hours, worms are washed off the plates and approximately 25 individuals added to each well of a 96-well microtitre plate and then assayed for their survival. Compounds or extracts that extended worm survival by 2- to 3fold were selected for further analyses. While this screen was carried out manually, the availability of the Union Biometrica Biosort (http://www.unionbio.com/products/copas2.html) allows automation of the different steps.

\section{Figure 2}

Hypothetical host-pathogen two dimensional array inspired by data from Benghezal et al. [*10]. The ability of host mutants to resist (green) or to be killed (red) by different bacterial strains and bacterial mutants is indicated. Gene names are arbitrary with $h r g$ and $p v f$ for Host Resistance Gene and Pathogen Virulence Factor, respectively. Based on this matrix, it can be speculated that $h r g-1$ encoded protein is specifically involved in a mechanism necessary for host resistance to bacterial virulence factors encoded by $p v f B$ and $p v f C$. The hrg-3-pvfD interaction would correspond to the case described by Liehl and colleagues [**18] with hrg-3 and $p v f D$ being the Drosophila Imd and Pseudomonas aprA genes, respectively. Finally, HRG-2 and HRG-4 can be host proteins necessary for bacterial invasion by pathogen $\mathrm{C}$ and pathogen $\mathrm{B}$ and $\mathrm{C}$, respectively, corresponding to the observations described in the reports by Philips et al. and Agaisse et al. [*12, *13]. 


\section{ACKNOWLEDGMENTS}

We thank Pierre Golstein for helpful criticism. Work in the authors' laboratory is supported by the Fondation Recherche Médicale, INSERM, the CNRS, the French Ministry of Research, Marseille-Nice Génopole, the Réseau Nationale des Génopoles, the European Union and the ANR. 
Figure 1

Day 0

50 eggs on growth plate

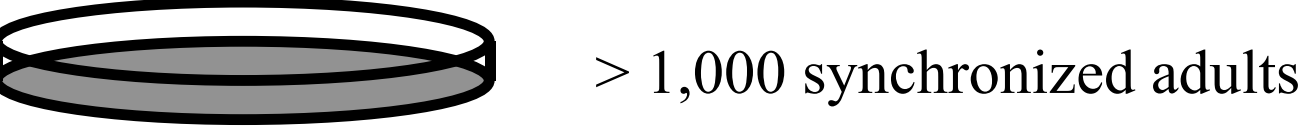

Day 6

Day 6.5

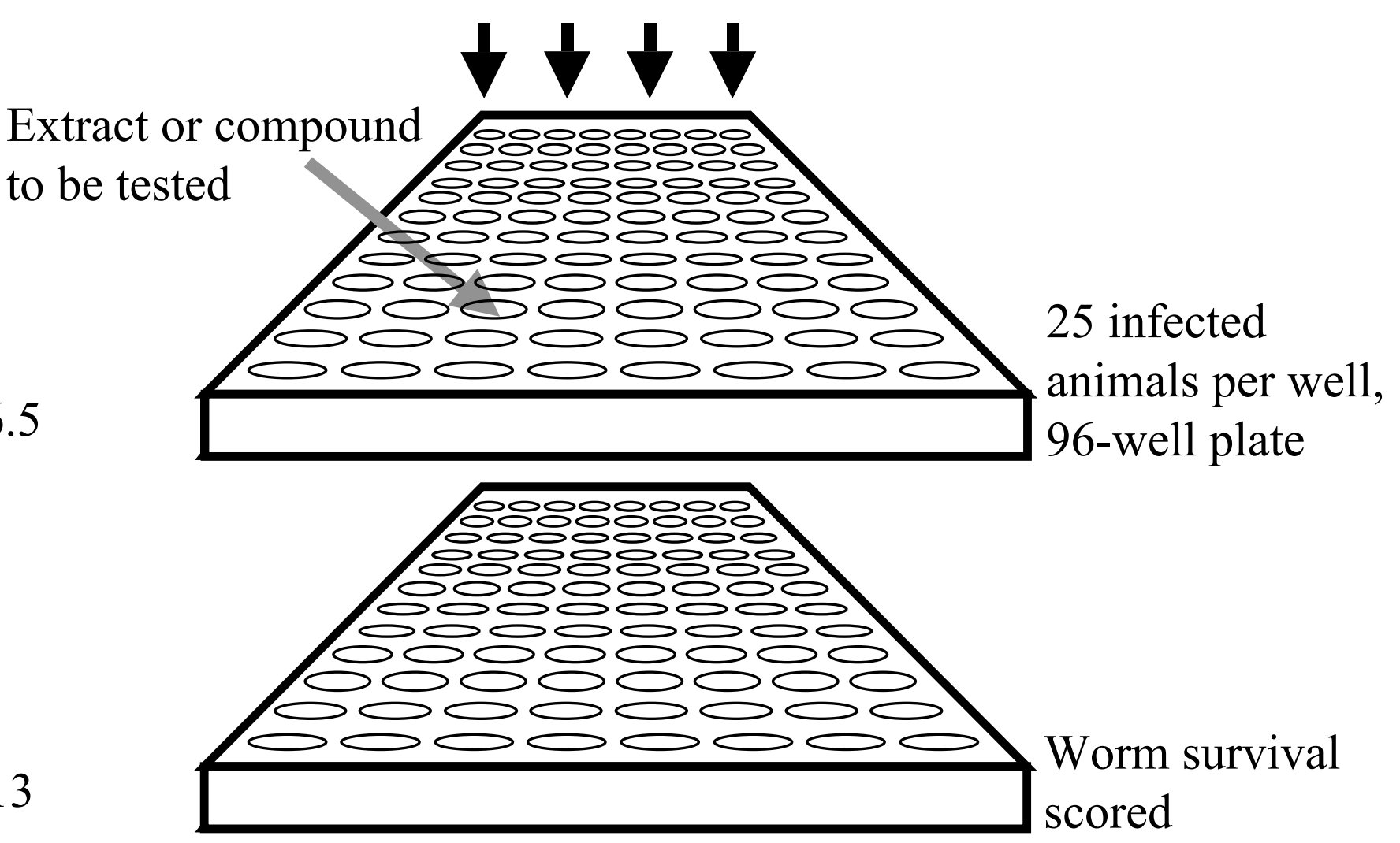


Figure 2

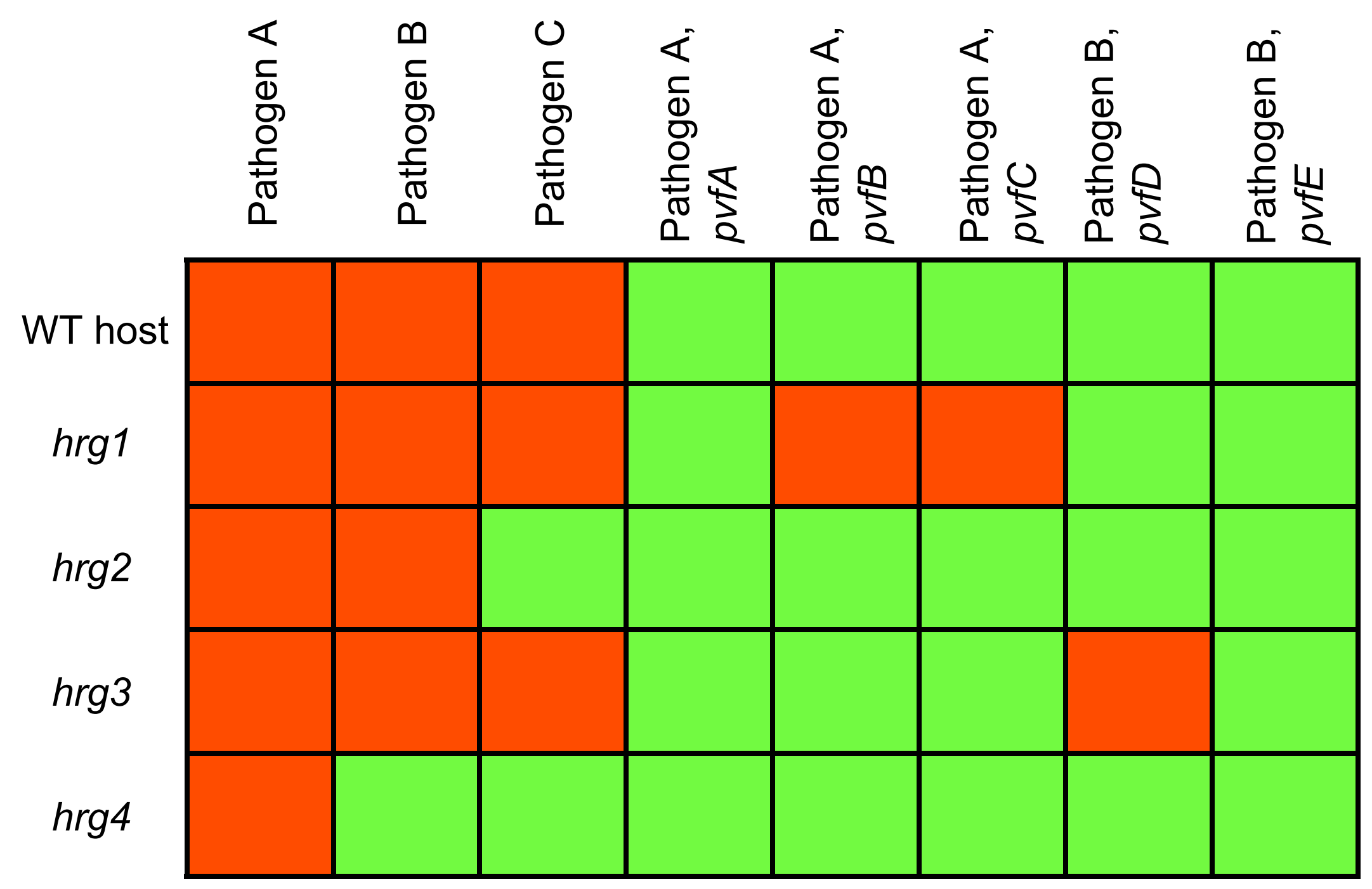

